\title{
Multivariate genome-wide association analysis identifies novel and relevant variants associated with anterior cruciate ligament rupture risk in the dog model
}

Lauren A. Baker', Guilherme J. M. Rosa², Zhengling Hao ${ }^{1}$, Alexander Piazza', Christopher Hoffman', Emily E. Binversie ${ }^{1}$, Susannah J. Sample ${ }^{1}$ and Peter Muir ${ }^{1 *}$ (i)

\begin{abstract}
Background: Anterior cruciate ligament rupture (ACLR) is a debilitating and potentially life-changing condition in humans, as there is a high prevalence of early-onset osteoarthritis after injury. Identification of high-risk individuals before they become patients is important, as post-treatment lifetime burden of ACLR in the USA ranges from \$7.6 to $\$ 17.7$ billion annually. ACLR is a complex disease with multiple risk factors including genetic predisposition. Naturally occurring ACLR in the dog is an excellent model for human ACLR, as risk factors and disease characteristics in humans and dogs are similar. In a univariate genome-wide association study (GWAS) of 237 Labrador Retrievers, we identified 99 ACLR candidate loci. It is likely that additional variants remain to be identified. Joint analysis of multiple correlated phenotypes is an underutilized technique that increases statistical power, even when only one phenotype is associated with the trait. Proximal tibial morphology has been shown to affect ACLR risk in both humans and dogs. In the present study, tibial plateau angle (TPA) and relative tibial tuberosity width (rTTW) were measured on bilateral radiographs from purebred Labrador Retrievers that were recruited to our initial GWAS. We performed a multivariate genome wide association analysis of ACLR status, TPA, and rTTW.
\end{abstract}

Results: Our analysis identified 3 loci with moderate evidence of association that were not previously associated with ACLR. A locus on Chr1 associated with both ACLR and rTTW is located within ROR2, a gene important for cartilage and bone development. A locus on Chr4 associated with both ACLR and TPA resides within DOCK2, a gene that has been shown to promote immune cell migration and invasion in synovitis, an important predictor of ACLR. A third locus on Chr23 associated with only ACLR is located near a long non-coding RNA (IncRNA). LncRNA's are important for regulation of gene transcription and translation.

Conclusions: These results did not overlap with our previous GWAS, which is reflective of the different methods used, and supports the need for further work. The results of the present study are highly relevant to ACLR pathogenesis, and identify potential drug targets for medical treatment.

Keywords: ACL rupture, CCL rupture, GWAS, Complex trait, Canine, Bayesian approach

\footnotetext{
* Correspondence: peter.muir@wisc.edu

${ }^{1}$ Comparative Orthopaedic Research Laboratory, School of Veterinary

Medicine, University of Wisconsin-Madison, 2015 Linden Drive, Madison,

Wisconsin, USA

Full list of author information is available at the end of the article
}

(c) The Author(s). 2018 Open Access This article is distributed under the terms of the Creative Commons Attribution 4.0 International License (http://creativecommons.org/licenses/by/4.0/), which permits unrestricted use, distribution, and reproduction in any medium, provided you give appropriate credit to the original author(s) and the source, provide a link to the Creative Commons license, and indicate if changes were made. The Creative Commons Public Domain Dedication waiver (http://creativecommons.org/publicdomain/zero/1.0/) applies to the data made available in this article, unless otherwise stated. 


\section{Background}

Anterior cruciate ligament rupture (ACLR) is common in human beings, particularly in young, athletic individuals [1]. ACLR is a debilitating injury with a long recovery period. There is a high prevalence of early-onset knee osteoarthritis after injury [2]. Treatment of ACLR is costly; the lifetime burden of ACLR in the US is estimated at $\$ 7.6$ billion annually when treated with surgical reconstruction and $\$ 17.7$ billion annually if treated with rehabilitation [3]. Nearly three quarters of these cases can be classified as non-contact ruptures, typically occurring during landing or pivoting movements [4]. A clear explanation for ACLR without physical contact is not yet available, though it is generally understood that non-contact ACLR is a complex disease caused by a combination of intrinsic (variables that describe the individual) and extrinsic (variables that describe the environment of the individual) risk factors $[4,5]$.

Identification of intrinsic factors that increase individual risk of ACLR is an important epidemiologic goal. Thorough understanding of these risk factors will provide physicians with the means to identify individuals at high risk before they become patients in the orthopaedic surgeon's office. Young women are up to $10 \times$ more likely to rupture their ACL compared to men [6]. Among these women, a higher than average body mass index is a significant risk factor [7, 8]. Gonadal hormones may also play a role in ACLR risk [8]. Certain characteristics of femoral and tibial morphology have also been implicated in ACLR pathophysiology. The general hypothesis underlying these risk factors is that bone morphology can alter knee joint biomechanics, potentially placing excessive stress on the ACL. For instance, it has been suggested that a narrow femoral intercondylar notch may impinge on the ACL in certain knee positions [5]. Increased posterior tibial slope (PTS) may increase anterior-directed forces on the tibia leading to greater weight-bearing load on the ACL that in certain situations may exceed its failure strength $[5,9]$.

Genetics plays an important and deterministic role in directing development of individual morphology and physiology. Therefore, it is perhaps unsurprising that familial analyses support the existence of genetic influence on ACLR risk. A close family history of ACLR doubles individual risk [10] and increases the risk of ACL graft rupture and contralateral ACLR $[11,12]$. Candidate gene studies have reported variants in genes for collagens, proteoglycans, matrix metalloproteinases, angiogenesis-associated proteins, elastin, and fibrillin [13]. A recent genome-wide association study (GWAS) in humans did not identify any variants that met genome-wide significance but had a small case sample size of 598 individuals of various ethnic backgrounds [14]. Additionally, much of the existing research on ACLR has come from a single population, and further research is needed to confirm or deny reported associations [13, 15]. Because ACLR is a complex disease, the genetic influence on ACLR is likely composed of many variants in multiple genomic loci with small to moderate individual effects [16]. Discovery of these smaller effect loci in human populations will require a combination of very large sample sizes, often in the hundreds of thousands, high-quality phenotyping, and sophisticated statistical methods [16].

One approach to identifying genetic variants that influence complex diseases is to study the trait in a model organism that may improve GWAS utility, such as the dog. For many diseases, presenting clinical signs, pathogenesis, and treatment are extremely similar between humans and their canine counterparts [17]. The advantage of the dog lies in its unique history and genomic architecture [18]. Purebred dogs are closed populations descendent from a small number of founder individuals [18]. Selective breeding for visual and behavioral characteristics has also inadvertently selected for heritable diseases $[17,18]$. This selective process has created long regions of DNA in linkage disequilibrium (LD) that likely harbor disease risk variants [18]. This combined effect allows GWAS to be performed using fewer SNP markers and smaller sample sizes than would be required to perform the same experiment in human populations. Discoveries made in the dog can then be used to inform candidate gene studies in human populations, saving great effort in the way of time and research funding.

The canine knee joint is a long-established model for human knee pathology [19]. Though there are important differences regarding weight-bearing, gait, and joint range of motion in quadrupedal mammals, studies have shown the relative dimensions of internal knee structures, including the cruciate ligaments, are similar between dogs and humans, and the canine ACL has similar cell density, blood vessel density, and cell shape compared to the human ACL [20]. ACLR is the most common cause of pelvic limb lameness in the dog [21]. Like human ACLR, the vast majority of ruptures do not involve contact injury, typically occurring while the dog is running or playing in view of the owner. As in humans, multiple risk factors have been implicated, including influence of gonadal hormones [22-24] and high body condition score $[25,26]$. Anatomic risk factors that are associated with ACLR in dogs include narrow femoral intercondylar notch [27-29] distal femoral torsion [30], excessive tibial plateau angle (analogous to PTS in human beings) [31-34] and a relatively narrow tibial tuberosity width [35]. The most important risk factor for disease initiation is genetic influence, i.e. breed. While many breeds may be affected with ACLR, Newfoundlands, Rottweilers, and Labrador Retrievers are at especially high risk $[23,36]$. Heritability of ACLR 
in dogs is moderate at 0.3-0.5 [37-39]. Variants associated with ACLR in collagen genes have been reported [40]. A GWAS in the Newfoundland breed identified variants in genes associated with neuronal signaling pathways [41]. Through linear mixed model GWA analysis, the present authors identified 128 SNPs in 99 regions that were associated with ACLR in the Labrador Retriever [39]. Gene set and pathway analysis identified enrichment for genes associated with angiogenesis, innate immune mechanisms, and extracellular matrix proteins, particularly aggrecan, which has been linked to ACLR in humans [42, 43].

Given the complex, polygenic nature of ACLR $[39,41]$, it is likely that additional variants remain to be identified. InPower analysis [44] of our Labrador Retriever discovery GWAS suggested that at least 172 loci influence ACLR risk in the dog [39]. While improved statistical power is most often achieved through larger sample sizes, joint analysis of multiple correlated phenotypes is an underutilized technique that has the potential to increase statistical power to detect the moderate and small effect associations expected with complex traits without the logistical concerns associated with increasing sample size [16]. To date, published GWA analyses in the dog model have all been univariate, i.e. they consider each phenotype independently. Here we present a multivariate genetic association analysis of anatomic variables, tibial plateau angle (TPA) and relative tibial tuberosity width (rTTW), with ACLR case-control status in the dog model. We identified three loci with moderate association, two of which reside in genes that have not been previously linked to ACLR but have an established role in chronic immune-mediated conditions of human beings, including rheumatoid arthritis and progression of osteoarthritis. Both of these genes have been identified as drug targets for other disorders and may be candidates for ACLR treatment.

\section{Methods}

Recruitment

Recruitment and quality control have been reported previously [39]. Purebred Labrador Retriever dogs were recruited through the University of Wisconsin-Madison UW Veterinary Care Hospital, online advertising, and contact with local and national breed clubs. If available, a pedigree was collected from each dog enrolled in the study to confirm purebred status. The Labrador Retriever is well-suited to GWAS because it is relatively outbred compared to some breeds with smaller population size, and has an average LD decay over distance of $20 \mathrm{~Kb}$ [45]. This allows for a higher mapping resolution than in breeds with LD decay over distance of $>1 \mathrm{Mb}$. Full siblings were excluded from the analysis to avoid bias due to over-represented genotypes in closely related individuals [46]. Control dogs were over 8 years of age
[47], had stable stifles on examination, and no signs of effusion or osteophytosis on lateral radiographs [48]. Affected dogs were of any age, with examination and radiographic signs consistent with ACLR [49].

\section{Genotyping}

Blood or saliva was collected from case and control dogs for DNA extraction. Dogs were genotyped using the Illumina Canine HD BeadChip, which contains approximately $230 \mathrm{~K}$ single nucleotide polymorphism (SNP) markers on the CanFam3.1 reference genome. Quality control was performed using PLINK [50] and a previously established protocol [39]. Briefly, SNPs were removed for minor allele frequency $<0.01$, missingness $>90 \%$, and Hardy-Weinberg equilibrium of $P<1 \mathrm{E}-7$. All individuals had a genotyping call rate of $>95 \%$.

\section{Radiographic Knee Morphology}

Bilateral knee radiographs were evaluated to confirm that the limb was not appreciably rotated before measurement. The position of the proximal tibia relative to the proximal fibula was evaluated to assess the degree of tibial rotation along the long axis of the bone. Radiographs with substantial rotational malpositioning were removed from the analysis. TPA and rTTW were measured using Horos DICOM image viewing and measurement tools (http://www.horosproject.org). These traits are stable in skeletally mature animals. Measurements were made according to published techniques [35, 51] (Fig. 1). If bilateral radiographs were available and of adequate quality, TPA and rTTW were measured bilaterally and the final value was the average of the two measurements.

\section{Statistical analysis}

To avoid losing dogs due to missing information, dogs with unknown weight ( $n=7$ cases, 30 controls) or age ( $n=5$ cases, 4 controls) were assigned the average weight or age of their case or control group before radiographic evaluation. For cases, this weight was 37.05 kilograms and age was 5.95 years. For controls, this weight was 34.16 kilograms and age was 10.53 years. Age, weight, TPA and rTTW data were tested for normality using the Shapiro-Wilk test. Measured variables were evaluated independently for differences between cases and controls using the Wilcoxon rank-sum test. Data summaries and initial statistical analyses were performed using the $\mathrm{R}$ statistical package [52]. To correct for population structure and confounding variables in the dataset, principal components of a genetic relationship matrix were estimated from a pruned set of SNPs with LD $\mathrm{r}^{2}<0.5$. Catell's scree test was used to determine the number of principal components to retain for analysis (see Additional file 1) [53]. Case-control phenotypes were 


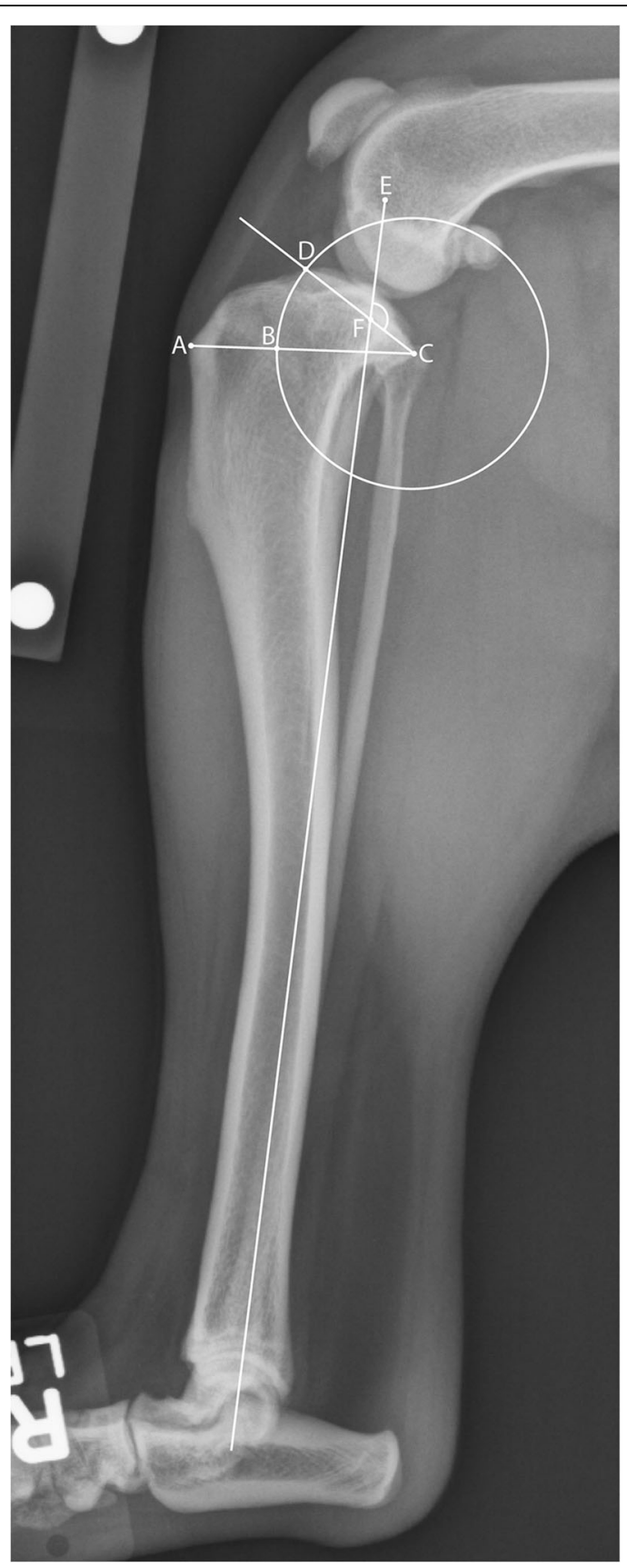

Fig. 1 Measurements of proximal tibial morphology taken from a lateral stifle radiograph. Before measurement, the radiograph was evaluated for appropriate technique and position. A. The most anterior point of the tibial tuberosity. B. The cross point of a circle on line AC where the center is at the most posterior point of the tibial plateau (C) and also crosses the most anterior point of the tibial plateau (D). C. The most posterior point of the tibial plateau. D. The most anterior point of the tibial plateau. E. A reference line extending from the center of the intercondylar eminence proximally to the center of the tarsus distally. $F$. The cross point of line $E$ and the tibial slope line (DC). Tibial plateau angle (TPA) was measured as the obtuse angle at EFC - $90^{\circ}$. Relative tibial tuberosity width ( $r T T W$ ) was measured as the width of the tibial tuberosity divided by the width of the proximal tibia not including the tibial tuberosity $(\mathrm{AB} / \mathrm{BC})$ residuals of multiple logistic regression against variables known to influence ACLR in dogs [23]: age, weight, and neuter status, as well as the first 6 principal components. Before association analysis, each phenotype was quantile transformed to a standard normal distribution [54]. Genome-wide multivariate association was performed using mvBIMBAM software $[54,55]$. MvBIMBAM frames association analysis as a model comparison problem whereby genotypes may be directly associated with a phenotype, indirectly associated with a phenotype (perhaps through another phenotype), or unassociated with a phenotype. The genotype may also be directly associated with more than one phenotype (referred to here as the multivariate phenotype). Bayes Factors are calculated to measure support for each model compared to the null (no association). The model comparison framework allows the user to determine not only whether an association exists between a genotype and phenotype(s), but which phenotypes are responsible for the association [55]. Bayes Factors ( $\log _{10}$ scale) were evaluated for evidence of association with the multivariate phenotype. Marginal posterior probabilities of associated SNPs were then evaluated to determine which phenotypes may be influencing the association. Regions with evidence of association were evaluated using the CanFam 3.1 Broad Improved Canine Annotation catalog [56] in the UCSC Table Browser [57].

\section{Results}

Genotyping information was available for 336 purebred Labrador Retriever dogs. 114 dogs were excluded due to radiographs of inadequate quality, leaving 222 dogs for GWAS analysis. The final dataset contained 135,482 SNPs from 69 cases and 153 controls. The ratio of females to males in case and control groups was 0.97 and 0.88 , respectively. Of the 116 males, 83 were castrated (71.5\%). Of the 106 females, 87 were ovariohysterectomized $(82.1 \%)$. There was no significant difference in the distribution of neutered animals across case and control groups $\left(X^{2}=0.1583, P=0.69\right)$.

Before missing data imputation, average ages of case and control dogs retained for analysis were 6.4 and 10.5 years, respectively, and average weights were 35.7 and $35.3 \mathrm{~kg}$, respectively. A summary of statistical analysis for individual covariates and additional phenotypes is given in Table 1. Shapiro-Wilk tests confirmed all variables were not normally distributed (see Additional files 2 and 3). Therefore, Wilcoxon rank-sum tests were used to evaluate variables for differences between case and control groups. By design, dogs in the control group were significantly older than those in the case group. Dogs affected with ACLR were both significantly heavier and had a 
Table 1 Summary statistics for individual covariates and phenotypes in ACLR case and control groups

\begin{tabular}{llll}
\hline Variables & Case & Control & $P$ \\
\hline Age (years) & $6.4(0.88-12.5)$ & $10.5(8.0-14.8)$ & $<2.2 \mathrm{E}-16^{*}$ \\
Weight (kg) & $37.1(27.0-58.5)$ & $34.2(20.8-50.3)$ & $0.00015^{*}$ \\
TPA (degrees) & $29.0(20.9-35.0)$ & $28.0(20.9-37.6)$ & 0.13 \\
rTTW & $0.64(0.46-1.00)$ & $0.70(0.45-1.00)$ & $0.00032^{*}$
\end{tabular}

All values reflect median values and range (parentheses) in case and control datasets. Values with an asterisk $\left(^{*}\right)$ indicate that the result was significant Abbreviations: TPA tibial plateau angle, $r T T W$ relative tibial tuberosity width $P$-values are the result of Wilcoxon rank-sum tests performed for age, weight, TPA, and rTTW

significantly smaller rTTW than unaffected dogs. Measured TPA did not differ between case and control groups.

The multivariate GWAS provided moderate evidence of association ( $\log _{10}$ Bayes Factor $>3$ ) for 4 SNPs with the phenotypes (Fig. 2, Table 2). Of these, two SNPs on chromosome 23 were in perfect LD and may be considered a single association. The three regions identified were on chromosomes 1, 4, and 23. The association on chromosome 1 is located within the tyrosine-protein kinase transmembrane receptor 2 gene (ROR2), which is involved in early formation of chondrocytes, and is required for cartilage and growth plate development. The association on chromosome 4 is within the dedicator of cytokinesis 2 gene (DOCK2), an important regulator of lymphocyte migration. The association on chromosome 23 is in an intergenic region close to a long non-coding RNA of unknown function.

An advantage of the mvBIMBAM package is the ability to discern which phenotypes in the multivariate model are responsible for the association. The posterior probability of effect (PPE) calculated for each SNP and the corresponding phenotype are displayed in Table 3. All identified SNPs showed strong probability of association with ACLR. In addition to association with the case-control phenotype, the DOCK2 SNP was also associated with TPA and the ROR2 SNP was also associated with rTTW. The association on chromosome 23 was primarily associated with ACLR and not TPA or rTTW. Evidence for this can be seen with higher $\mathrm{BF}_{\mathrm{ACLR}}$ compared to $\mathrm{BF}_{\text {mult }}$ and relatively low PPE of TPA and rTTW compared to ACLR for this locus. It should be noted that PPE is the sum of the probability of a direct effect and the probability of an indirect effect on the phenotype. In all cases, the PPE was primarily explained by the probability of a direct effect on the individual phenotypes, with little influence from indirect effect.

\section{Discussion}

Through multivariate association analysis of ACLR case and control dogs, TPA, and rTTW, we have identified three loci with moderate evidence of association with ACLR in the Labrador Retriever model. Two of these loci are within genes that have not previously been linked to ACLR in human or dog studies, though they are highly relevant given what is known regarding ACLR pathogenesis. ACLR in dogs is a highly polygenic complex trait $[39,41]$, and our group previously reported 99 risk loci associated with the condition using a dataset containing many of the same dogs used in the current analysis [39]. The results of the current study should be considered complimentary and provides evidence for the benefit of using a multivariate approach to improve power to detect smaller effect associations expected when studying the genetic contribution to complex trait disease.

Before association analysis, we evaluated covariates and supplementary phenotypes for differences between cases and controls. The statistically significant difference in age between cases and controls was expected, as age was an important part of the recruitment strategy [39]. Additionally, case dogs in the sampled group weighed significantly more than controls. The association between higher body weight and increased risk of ACLR in dogs has been reported $[35,58]$. While this is a notable finding, it is important to recognize that these findings

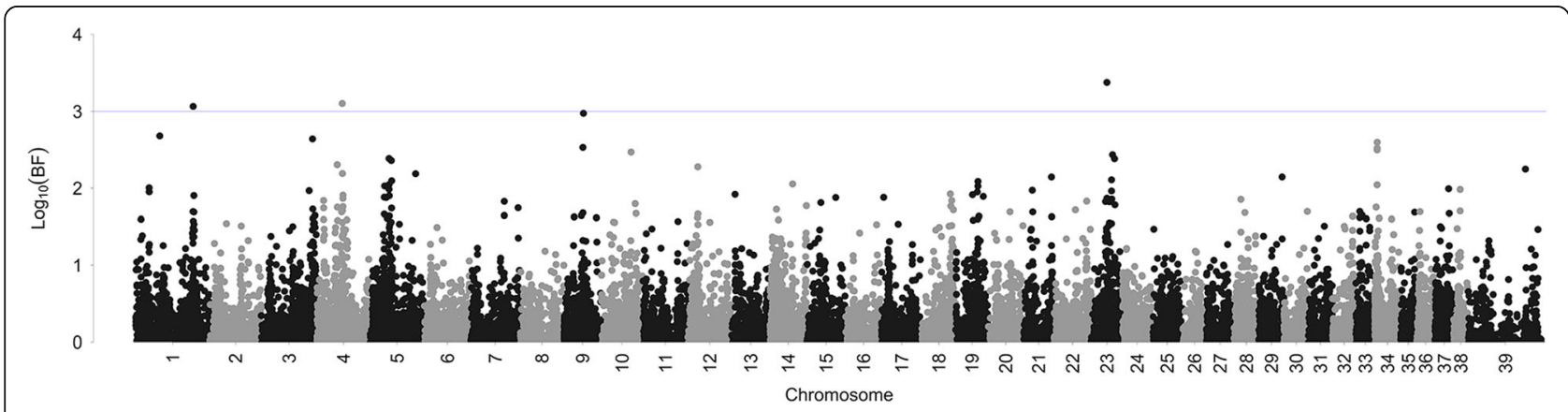

Fig. 2 Manhattan plot of $\log _{10}$ Bayes factors (BF) of the multivariate phenotype. The multivariate phenotype is the combined effect of tibial plateau angle (TPA), relative tibial tuberosity width ( $r$ TTW), and ACLR case-control status. SNPs on chromosomes 1, 4, and 23 showed moderate evidence of association with the multivariate phenotype (BF > 3) 
Table 2 SNPs associated with multivariate phenotype of anterior cruciate ligament rupture (ACLR), tibial plateau angle (TPA), and relative tibial tuberosity width (rTTW)

\begin{tabular}{llllllllll}
\hline SNP & Chr & Position & BF $_{\text {mult }}$ & BF $_{\text {ACLR }}$ & BF $_{\text {TPA }}$ & BF $_{\text {rTTw }}$ & Gene & Location & Exon Dist. (Kb) \\
\hline BICF2G630788965 & 1 & 95183740 & 3.07 & 0.78 & -0.23 & 1.75 & ROR2 & $95124036-95282085$ & 51.9 \\
BICF2P1286728 & 4 & 42104780 & 3.10 & 0.68 & 2.18 & -0.24 & DOCK2 & $41779969-42177966$ & 0.34 \\
BICF2P1160758 & 23 & 26140588 & 3.37 & 4.24 & -0.16 & -0.11 & (IncRNA) & $26107359-26132719$ & N/A \\
TIGRP2P303751_rs8873754 & 23 & 26148612 & & & & & & & \\
\hline
\end{tabular}

Table shows $\log _{10}$ Bayes Factors $(\mathrm{BF})$ for multivariate association test as well as univariate tests. $\mathrm{BF}_{\text {mult }}$, result for multivariate phenotype; $\mathrm{BF}_{\mathrm{ACLR}}, \mathrm{ACL}$ rupture only; $\mathrm{BF}_{\mathrm{TPA}}$, tibial plateau angle only; $\mathrm{BF}_{\mathrm{rTTw}}$, relative tibial tuberosity width only. SNPs on chromosomes 1 and 4 were located within intronic regions of genes. The distance from the SNP location to the nearest gene exon is reported (Exon Dist.). The locus on chromosome 23 resides in a region $<8 \mathrm{~Kb}$ from a long non-coding RNA (IncRNA)

do not distinguish between dogs of large body size and those that are overweight.

Several studies have evaluated the effect of excessive TPA on ACLR risk in dogs. The ACL opposes anterior tibial thrust. A steeper TPA increases anterior tibial thrust, placing increased mechanical stress on the ACL and potentially predisposing the ligament to rupture [47]. Results of case control studies of TPA on ACLR risk have been inconsistent with multiple studies declaring an excessively steep TPA as a risk factor [31-34] while others were not able to identify a significant effect $[47,59,60]$. The present study did not identify a difference in TPA between affected and unaffected dogs. However, the TPA of affected dogs was steeper than that of unaffected dogs, which is consistent with previous studies that were able to identify significant differences. Though it remains plausible that TPA has some effect on ACLR risk in dogs, it is likely that this effect is either indirect or fairly small. PTS in humans is analogous to TPA measured in dogs. Similar to dogs, human patients affected with ACLR have steeper PTS [61-63] and this effect may be more pronounced in female patients [64].

Evaluation of rTTW and ACLR status confirmed results of a previous study that reported the rTTW of dogs affected with ACLR is significantly narrower than the rTTW of unaffected dogs. Dogs with a relatively narrow tibial tuberosity experience greater anterior tibial thrust, again placing greater mechanical stress on the ACL and potentially increasing rupture risk. While our study was able to confirm rTTW as a risk factor for ACLR in dogs, a recent study in small breed dogs did not identify a difference between case and control groups [34]. It may be that risk due to rTTW is limited to large breed dogs,

Table 3 The posterior probability of effect (PPE) calculated for each SNP and corresponding phenotype

\begin{tabular}{llll}
\hline SNP & PPE $_{\text {ACLR }}$ & PPE $_{\text {TPA }}$ & PPE $_{\text {rTTW }}$ \\
\hline BICF2G630788965 & 0.87 & 0.28 & 0.99 \\
BICF2P1286728 & 0.86 & 0.99 & 0.48 \\
BICF2P1160758 & 0.99 & 0.49 & 0.54 \\
TIGRP2P303751_rs8873754 & & & \\
\hline
\end{tabular}

Posterior probability of effect (PPE) is 1 minus the probability of no effect on the phenotype and this possibility should be evaluated in future epidemiological studies. To the authors' knowledge, tibial tuberosity morphology has not been evaluated as a risk factor for ACLR in human beings.

The locus with greatest evidence of association was located on chromosome 23 . This locus was primarily associated with ACLR and not TPA or rTTW. This locus lies within an intergenic haplotype block that is less than $8 \mathrm{~Kb}$ from the transcription location of a long non-coding RNA (lncRNA) with unknown function. LncRNAs are broadly defined as long transcripts $(>200$ nucleotides) that do not function in a protein-coding fashion [65]. While some of these transcripts are likely non-functional, a growing list of lncRNAs have specific biological roles in many categories including cellular proliferation, chromatin remodeling, and regulation of gene transcription and translation $[65,66]$. LncRNA expression tends to be highly tissue-specific [67]. The lncRNA we identified appears to be primarily expressed in testicular tissue, but not ovarian tissue [56]. Expression levels were not evaluated in tissues associated with joints (e.g. collagenous tissue or synovium). While there is evidence of gonadal influence on ACLR risk in both humans and dogs $[23,24,68,69]$, it is difficult to speculate on potential function of this lncRNA, if any, without further investigation.

The SNP association located on chromosome 4 was within the DOCK2 gene. DOCK2 protein interacts with Rac1 to induce lymphocyte migration into tissues, including the synovium. DOCK2 signaling has been linked to synovitis associated with rheumatoid arthritis in human beings [70]. Lymphoplasmacytic synovitis precedes ACLR in dogs, and arthritis is typically present at the time of diagnosis [71, 72]. Additionally, numbers of lymphocytes are positively correlated with radiographic evidence of degenerative joint disease, indicating that lymphocytic inflammation likely contributes to progression of joint disease [73]. In humans, synovitis and associated lymphocytic inflammation plays a role in post-injury osteoarthritis progression and chronicity [74]. In a rabbit model of joint pathology, experimentally induced synovitis resulted in weakening of the ACL [75]. In both humans and dogs, surgical correction of joint 
instability fails to prevent osteoarthritis development and progression, indicating that disease progression has a substantial biochemical component [76-78]. It is feasible that aberrant DOCK2 signaling may contribute to the inflammatory cascade associated with ACLR and play a role in both onset and progression of disease.

The SNP association located on chromosome 1 was within the ROR2 gene, a protein with multiple roles, any of which may be relevant to ACLR. ROR2 has a well-established role in bone development, where it is essential for appropriate patterning and chondrocyte expansion during growth [79]. ROR2 also plays complimentary roles in both osteoblastogenesis and osteoclastogenesis from mesenchymal stem cells (MSCs) [80, 81]. Aberrant ROR2 signaling appears to play a role in bone loss associated with rheumatoid arthritis [82] and has been shown to be significantly up-regulated in these patients [83]. ROR2 has a more general role in cellular proliferation and migration, and this role has been implicated in several cancers of various tissue types [84, 85]. Its role in cellular expansion also has important implications for joint healing and homeostasis [79] as tissue repair requires proliferation and migration to sites of healing. Down-regulation of ROR2 inhibits the regenerative capacity of chondrocytes [79]. Indeed, ROR2 is down-regulated in human patients with end-stage osteoarthritis [80]. These roles indicate that altered ROR2 signaling during development may affect the patterning and growth of long bones in a way that augments ACLR risk. Later in life, aberrant ROR2 signaling may influence processes important for healing damaged ligament or cartilage and could contribute to post-rupture progression of osteoarthritis.

The results of the current study did not overlap with those from our previous GWAS of ACL rupture in Labrador Retrievers [39]. The present analysis was substantially different from our previous GWAS in a number of ways, and this is likely to have impacted the results. The current dataset represents dogs that had been in the previous GWAS as well as dogs that have since been added to our dataset. Therefore, while dog breed, geographical location, and recruitment strategy remained constant, the sample analyzed was substantially different from the previous analysis. Additionally, we made further correction for environmental risk factors by regressing the case-control phenotype against age, weight, and neutered status, whereas the previous study only included neutered status as a covariate. It should also be noted that the present multivariate analysis was performed using an algorithm that employs a Bayesian approach, while the previous GWAS was based on traditional linear mixed model analysis of a single case-control phenotype. It is not clear at this point how much additional variance may be explained by the variants discovered in the present study. Accurate calculation of SNP heritability $\left(h_{\mathrm{SNP}}^{2}\right)$ and heritability explained by a subset of genome-wide significant SNPs in a relatively inbred species with known relatedness is potentially complex, and will certainly require an independent sample at least as large or larger than the dataset used for this analysis [86]. Ultimately, the difference in results between the two studies highlights the need for validation in a new and larger population, as well as through other methods beyond GWAS including whole genome sequencing and gene expression studies.

\section{Conclusions}

A multivariate analysis of ACLR, rTTW, and TPA has identified three novel variants with moderate probability of effect on ACLR. Two of these variants reside within genes that have previously been implicated in other joint pathologies, particularly rheumatoid arthritis and progression of osteoarthritis. This analysis complements our previous discovery GWAS of ACLR in the dog model, and provides further support for ACLR as a complex, multifactorial disease that may be influenced by aberrant signaling in the immune system and/or developmental variables. While multiple GWAS for ACLR have been performed in the dog model, this study is the first to identify genes directly related to joint morphology and homeostasis, which speaks to the power of the multivariate approach. Both DOCK2 and ROR2 represent potential drug targets for therapies targeting the biochemical aspect of ACLR and associated osteoarthritis. CPYPP is a small molecule inhibitor of DOCK2 that may provide the scaffold for a DOCK2-targeting immunosuppressant [87]. As an identified oncogene, ROR2 has been described as a candidate for targeted therapy for several cancers, and the search for an effective ROR2 inhibitor is currently underway. Future work to validate these loci as bona fide risk factors for ACLR will provide improved understanding of ACLR pathogenesis as well as the opportunity for medical intervention through targeted drug therapy. Such advances will have an important impact on both human and animal orthopaedic health.

\section{Additional files}

Additional file 1: Scree plot of variance explained by each principal component. The first 6 principal components were used to account for the majority of variance in the dataset. (TIFF $87 \mathrm{~kb}$ )

Additional file 2: Distribution of tibial plateau angle (TPA) measurements among cases and controls. (TIFF $10280 \mathrm{~kb}$ )

Additional file 3: Distribution of relative tibial tuberosity width ( $r T T W$ ) among cases and controls. (TIFF $10280 \mathrm{~kb}$ )

\section{Abbreviations}

ACLR: Anterior Cruciate Ligament Rupture; GWAS: Genome-wide association study; TPA: Tibial plateau angle; rTTW: Relative tibial tuberosity width; ROR2: Tyrosine-protein kinase transmembrane receptor 2 gene; DOCK2: Dedicator of cytokinesis 2 gene; InCRNA: Long non-coding RNA; 
PTS: Posterior tibial slope; LD: Linkage disequilibrium; SNP: Single nucleotide polymorphism; BF: Bayes Factor; PPE: Posterior probability of effect; MSCs: Mesenchymal stem cells

\section{Acknowledgments}

The authors would like to thank the faculty, residents, and students throughout the University of Wisconsin-Madison UW Veterinary Care Hospital and the many individual breeders and pet owners for their help in recruitment of Labrador Retrievers for this study. The authors would also like to thank the American Kennel Club Canine Health Foundation, which provided support by giving access to the AKC pedigree database.

\section{Funding}

This work was supported by grants from the Morris Animal Foundation (D13CA-020, www.morrisanimalfoundation.org), the Robert Draper Technology Innovation Fund from the University of Wisconsin-Madison and the Melita Grunow Family Professorship in Companion Animal Health. Lauren Baker received support from the National Institutes of Health (T32OD10999) and is supported by a National Library of Medicine training grant to the UWMadison Computation and Informatics in Biology and Medicine Training Program (NLMT15LM007359). Susannah Sample received support from the National Institutes of Health (K01OD019743-01A1). The funders had no role in study design, data collection and analysis, decision to publish, or preparation of the manuscript.

\section{Availability of data and materials}

The datasets used and/or analyzed during the current study are available from the corresponding author on reasonable request.

\section{Authors' contributions}

$\angle A B$ assisted in data collection, performed all radiographic measurements, analyzed the data, and drafted the manuscript. GJMR provided support for statistical analysis and methods. ZH processed samples and performed DNA extraction for genotyping. AP, CH, EEB, SJS all assisted animal phenotyping and data collection. PM conceived of the idea for the project, supervised the project, provided grant funding, and finalized the manuscript. All authors have had the opportunity for critical evaluation and have approved of the final manuscript.

\section{Ethics approval and consent to participate}

All procedures were performed in strict accordance with the recommendations in the Guide for the Care and Use of Laboratory Animals of the National Institutes of Health and the American Veterinary Medical Association and with approval from the Animal Care Committee of the University of WisconsinMadison (protocol \#V1070, V005463). Written informed consent was obtained from each owner before participation in the study.

\section{Consent for publication}

Not applicable.

\section{Competing interests}

PM and LAB are named on a patent application "Methods to predict heritable canine non-contact cruciate ligament rupture", P150052US02.

\section{Publisher's Note}

Springer Nature remains neutral with regard to jurisdictional claims in published maps and institutional affiliations.

\section{Author details}

${ }^{1}$ Comparative Orthopaedic Research Laboratory, School of Veterinary Medicine, University of Wisconsin-Madison, 2015 Linden Drive, Madison, Wisconsin, USA. ${ }^{2}$ Department of Animal Sciences, College of Agricultural and Life Sciences, University of Wisconsin-Madison, 1675 Observatory Drive, Madison, Wisconsin, USA.

Received: 25 January 2018 Accepted: 13 June 2018

\section{Published online: 26 June 2018}

\section{References}

1. Sanders TL, Maradit Kremers H, Bryan AJ, Larson DR, Dahm DL, Levy BA, et al. Incidence of anterior cruciate ligament tears and reconstruction: a 21year population-based study. Am J Sports Med. 2016;44:1502-7.
2. Lohmander LS, Ostenberg A, Englund M, Roos H. High prevalence of knee osteoarthritis, pain, and functional limitations in female soccer players twelve years after anterior cruciate ligament injury. Arthritis Rheum. 2004; 50(10):3145-52.

3. Mather RC, Koenig L, Kocher MS, Dall TM, Gallo P, Scott D, et al. Societal and economic impact of anterior cruciate ligament tears. J Bone Joint Surg Am. 2013;95:1751-9.

4. Smith HC, Vacek P, Johnson RJ, Slauterbeck JR, Hashemi J, Shultz S, et al. Risk factors for anterior cruciate ligament injury: a review of the literature part 2: hormonal, genetic, cognitive function, previous injury, and extrinsic risk factors. Sports Health. 2012;4:155-61.

5. Smith HC, Vacek P, Johnson RJ, Slauterbeck JR, Hashemi J, Shultz S, et al. Risk factors for anterior cruciate ligament injury: a review of the literature part 1: neuromuscular and anatomic risk. Sports Health. 2012;4:69-78.

6. Gwinn DE, Wilckens JH, McDevitt ER, Ross G, Kao TC. The relative incidence of anterior cruciate ligament injury in men and women at the United States Naval Academy. Am J Sports Med. 2000;28:98-102

7. Uhorchak JM, Scoville CR, Williams GN, Arciero RA, Pierre PS, Taylor DC. Risk factors associated with noncontact injury of the anterior cruciate ligament. Am J Sports Med. 2003;31:831-42.

8. Shultz SJ, Schmitz RJ, Benjaminse A, Collins M, Ford K, Kulas AS. ACL Research Retreat VII: An Update on Anterior Cruciate Ligament Injury Risk Factor Identification, Screening, and Prevention: March 19-21, 2015; Greensboro, NC. J Athl Train. 2015;50:1076-93.

9. Hashemi J, Chandrashekar N, Gill B, Beynnon BD, Slauterbeck JR, Schutt RC, et al. The geometry of the tibial plateau and its influence on the biomechanics of the tibiofemoral joint. J Bone Joint Surg Am. 2008;90:2724-34.

10. Flynn RK, Pedersen CL, Birmingham TB, Kirkley A, Jackowski D, Fowler PJ. The familial predisposition toward tearing the anterior cruciate ligament: A case control study. Am J Sports Med. 2005;33:23-8.

11. Harner CD, Paulos LE, Geenwald AE, Rosenberg TD, Cooley VC. Detailed analysis of patients with bilateral anterior cruciate ligament injuries. Am J Sports Med. 1994;22:37-43.

12. Brophy RH, Schmitz L, Wright RW, Dunn WR, Parker RD, Andrish JT, et al. Return to play and future $\mathrm{ACL}$ injury risk after $\mathrm{ACL}$ reconstruction in soccer athletes from the Multicenter Orthopaedic Outcomes Network (MOON) group. Am J Sports Med. 2012;40:2517-22.

13. Kaynak M, Nijman F, van Meurs J, Reijman M, Meuffels DE. Genetic variants and anterior cruciate ligament rupture: a systematic review. Sports Med. 2017:47:1637-50.

14. Kim SK, Roos TR, Roos AK, Kleimeyer JP, Ahmed MA, Goodlin GT, et al. Genome-wide association screens for Achilles tendon and ACL tears and tendinopathy. PLoS One. 2017;12:e0170422.

15. John R, Dhillon MS, Sharma S, Prabhakar S, Bhandari M. Is there a genetic predisposition to cruciate ligament tear? A systematic review. Am J Sports Med. 2016;44:3262-9.

16. Robinson MR, Wray NR, Visscher PM. Explaining additional genetic variation in complex traits. Trends Genet. 2014;30:124-32.

17. Shearin AL, Ostrander EA. Leading the way: canine models of genomics and disease. Dis Model Mech. 2010;3:27-34.

18. Karlsson EK, Lindblad-Toh K. Leader of the pack: gene mapping in dogs and other model organisms. Nat Rev Genet. 2008;9:713.

19. Gregory MH, Capito N, Kuroki K, Stoker AM, Cook JL, Sherman SL. A reveiw of translational animal models for knee osteoarthritis. Arthritis. 2012;2012: 764621.

20. Proffen BL, McElfresh M, Fleming BC, Murray MM. A comparative anatomical study of the human knee and six animal species. Knee. 2012;19:493-9.

21. Comerford EJ, Smith K, Hayashi K. Update on the aetiopathogeneisis of canine cranial cruciate ligament disease. Vet Comp Orthop Traumatol. 2011;24:91-8.

22. Duerr FM, Duncan CG, Savicky RS, Park RD, Egger EL, Palmer RH. Risk factors for excessive tibial plateau angle in large-breed dogs with cranial cruciate ligament disease. J Am Vet Med Assoc. 2007;231:1688-91.

23. Witsberger TH, Villamil JA, Schultz LG, Hahn AW, Cook JL. Prevalence and risk factors for hip dysplasia and cranial cruciate ligament deficiency in dogs. J Am Vet Med Assoc. 2008;232:1818-24.

24. Torres de la Riva G, Hart BL, Farver TB, Overbauer AM, Messam LL, Willits N, et al. Neutering dogs: effects on joint disorders and cancers in golden retrievers. PLoS One. 2013:8:e55937.

25. Lampman TJ, Lund EM, Lipowitz AJ. Cranial cruciate disease: current status of diagnosis, surgery, and risk for disease. Vet Comp Orthop Traumatol. 2003;16:122-6. 
26. Adams P, Bolus R, Middleton S, Moores AP, Grierson J. Influence of signalment on developing cranial cruciate rupture in dogs in the UK. Small Anim Pract. 2011;52:347-52.

27. Aiken SW, Kass PH, Toombs JP. Intercondylar notch width in dogs with and without cranial cruciate ligament injuries. Vet Comp Orthop Traumatol. 1995:8:128-32

28. Comerford EJ, Tarlton JF, Avery NC, Bailey AJ, Innes JF. Distal femoral intercondylar notch dimensions and their relationship to composition and metabolism of the canine anterior cruciate ligament. Osteoarthritis Cartilage. 2006;14:273-8.

29. Lewis BA, Allen DA, Henrickson TD, Lehenbauer TW. Computer tomographic evaluation of the canine intercondylar notch in normal and cruciate deficient stifles. Vet Comp Orthop Traumatol. 2008;21:119-24.

30. Ragetly CA, Evans R, Mostafa AA, Griffon DJ. Multivariate analysis of morphometric characteristics to evaluate risk factors for cranial cruciate ligament deficiency in Labrador retrievers. Vet Surg. 2011;40:327-33.

31. Morris E, Lipowitz AJ. Comparison of tibial plateau angles in dogs with and without cranial cruciate ligament injuries. J Am Vet Med Assoc. 2001;218:363-6.

32. Mostafa AA, Griffon DJ, Thomas MW, Constable PD. Morphometric characteristics of the pelvic limbs of Labrador Retrievers with and without cranial cruciate ligament deficiency. Am J Vet Res. 2009;70:498-507.

33. Su L, Townsend KL, Au J, Wittum TE. Comparison of tibial plateau angles in small and large breed dogs. Can Vet J. 2015;56:610-4.

34. Janovec J, Kyllar M, Midgley D, Owen M. Conformation of the proximal tibia and cranial cruciate ligament disease in small breed dogs. Vet Comp Orthop Traumatol. 2017;30:178-83.

35. Inauen $\mathrm{R}$, Koch $\mathrm{D}$, Bass M, Haessig M. Tibial tuberosity conformation as a risk factor for cranial cruciate ligament rupture in the dog. Vet Comp Orthop Traumatol. 2009;22:16-20.

36. Whitehair JG, Vasseur PB, Willits NH. Epidemiology of cranial cruciate ligament rupture in dogs. J Am Vet Med Assoc. 1993;203:1016-9.

37. Wilke VL, Conzemius MG, Kinghorn BP, Macrossan PE, Cai W, Rothschild MF. Inheritance of rupture of the cranial cruciate ligament in Newfoundlands. J Am Vet Med Assoc. 2006;228:61-4.

38. Nielen AL, Knol BW, van Hagen MA, van der Gaag I. Genetic and epidemiological investigation of a birth cohort of boxers. Tijdscher Diergeneeskd. 2003;128:586-90.

39. Baker LA, Kirkpatrick B, Rosa GJ, Gianola D, Valente B, Sumner JP. Genomewide association analysis in dogs implicates 99 loci as risk variants for anterior cruciate ligament rupture. PLoS One. 2017;12:e0173810.

40. Baird AE, Carter SD, Innes JF, Ollier WE, Short AD. Genetic basis of cranial cruciate ligament rupture (CCLR) in dogs. Connect Tissue Res. 2014;55:27581

41. Baird AE, Carter SD, Innes JF, Ollier W, Short A. Genome-wide association study identifies genomic regions of association for cruciate ligament rupture in Newfoundland dogs. Anim Genet. 2014;45:542-9.

42. Mannion S, Mtintsilana A, Posthumus M, van der Merwe W, Hobbs H, Collins $M$, et al. Genes encoding proteoglycans are associated with the risk of anterior cruciate ligament ruptures. Br J Sports Med. 2014;48:1640-6.

43. Johnson JS, Morscher MA, Jones KC, Moen SM, Klonk CJ, Jacquet R, et al. Gene expression differences between ruptured anterior cruciate ligaments in young male and female subjects. J Bone Joint Surg Am. 2015;97:71-9.

44. Park J-H, Wacholder S, Gail MH, Peters U, Jacobs KB, Chanock SJ, et al. Estimation of effect size distribution from genome-wide association studies and implications for future discoveries. Nature Genet. 2010;42:570-5.

45. Gray MM, Granka JM, Bustamante CD, Sutter NB, Boyko AR, Zhu L, et al. Linkage disequilibrium and demographic history of wild and domestic canids. Genetics. 2009;181:1493-505.

46. Anderson CA, Pettersson FH, Clarke GM, Cardon LR, Morris AP, Zondervan KT. Data quality control in genetic case-control association studies. Nat Protoc. 2010;5:1564-73.

47. Reif $U$, Probst CW. Comparison of tibial plateau angles in normal and cranial cruciate deficient stifles of Labrador Retrievers. Vet Surg. 2004;32:385-9.

48. Chuang C, Ramaker MA, Kaur S, Csomos RA, Kroner KT, Bleedorn JA, et al. Radiographic risk factors for contralateral rupture in dogs with unilateral cranial cruciate ligament rupture. PLoS One. 2014;9:e106389.

49. Harasen G. Diagnosing rupture of the cranial cruciate ligament. Can Vet J. 2002:43:475-6.

50. Chang CC, Chow CC, Tellier LC, Vattikuti S, Purcell SM, Lee JJ. Secondgeneration PLINK: Rising to the challenge of larger and richer datasets. Gigascience. 2015;4:7.
51. Abel SB, Hammer DL, Shott S. Use of the proximal portion of the tibia for measurement of the tibial plateau angle in dogs. Am J Vet Res. 2003;64: 1117-23.

52. R Core Team. R: A language and environment for statistical computing. Vienna, Austria: R Foundation for Statistical Computing; 2016. URL http:// www.R-project.org

53. Cangelosi R, Goriely A. Component retention in principal component analysis with application to CDNA microarray data. Biol Direct. 2007;2:2.

54. Shim H, Chasman D, Smith JD, Mora S, Ridker P, Nickerson D, et al. A multivariate genome-wide association analysis of $10 \mathrm{LDL}$ subfractions, and their response to statin treatment, in 1868 Caucasians. PLoS One. 2015;10: e0120758.

55. Stephens M. A unified framework for association analysis with multiple related phenotypes. PLoS One. 2013;8:e65245.

56. Hoeppner MP, Lundquist A, Pirun M, Meadows JRS, Zamani N, Johnson J, et al. An improved canine genome and comprehensive catalogue of coding genes and non-coding transcripts. PLoS One. 2014:9:e91172.

57. Karolchik D, Hinrichs AS, Furey TS, Roskin KM, Sugnet CW, Haussler D, et al. The UCSC Table Browser data retrieval tool. Nucleic Acids Res. 2004;26: 2204-7.

58. Duval JM, Budsberg SC, Flo GL, Sammarco JL. Breed, sex, and body weight as risk factors for rupture of the cranial cruciate ligament in young dogs. J Am Vet Med Assoc. 1999:215:811-4.

59. Buote N, Fusco J, Radasch R. Age, tibial plateau angle, sex, and weight as risk factors for contralateral rupture of the cranial cruciate ligament in Labradors. Vet Surg. 2009;38:481-9.

60. Fuller MC, Kapatkin AS, Bruecker KA, Holsworth IG, Kass PH, Hayashi K. Comparison of the tibial mechanical joint orientation angles in dogs with cranial cruciate ligament rupture. Can Vet J. 2014;55:757-64.

61. Giffin JR, Vogrin TM, Zantop T, Woo SL, Sarner CD. Effects of increasing tibial slope on the biomechanics of the knee. Am J Sports Med. 2004;32:376-82.

62. Brandon ML, Haynes PT, Bonamo JR, Flynn MI, Barrett GR, Sherman MF. The association between posterior-inferior tibial slope and anterior cruciate ligament insufficiency. Arthroscopy. 2006;22:894-9.

63. Todd MS, Lalliss S, Garcia E, DeBerardino TM, Cameron KL. The relationship between posterior tibial slope and anterior cruciate ligament injuries. Am J Sports Med. 2010;38:63-7.

64. Hohmann E, Bryant A, Reaburn P, Tetsworth K. Is there a correlation between posterior tibial slope and non-contact anterior cruciate ligament injuries? Knee Surg Sports Traumatol Arthrosc. 2011;19:109-14.

65. Quinn JJ, Chang HY. Unique features of long non-coding RNA biogenesis and function. Nat Rev Genet. 2016;17:47-62

66. Kung JT, Colognori D, Lee JT. Long noncoding RNAs: past, present, and future. Genetics. 2013;193:651-69.

67. Cabili MN, Trapnell C, Goff L, Koziol M, Tazon-Vega B, Regev A, Rinn JL. Integrative annotation of human large intergenic noncoding RNAs reveals global properties and specific subclasses. Genes Dev. 2011;25:1915-27.

68. Beynnon BD, Shultz SJ. Anatomic alignment, menstrual cycle phase, and the risk of anterior cruciate ligament injury. J Athl Train. 2008;43:541-2.

69. Hewett T, Zazulak B, Myer G. Effects of the menstrual cycle on anterior cruciate ligament injury risk: a systematic review. Am J Sports Med. 2007;35: 659-68.

70. Whitaker JW, Boyle DL, Bartok B, Ball ST, Gay S, Wang W, et al. Integrative omics analysis of rheumatoid arthritis identifies non-obvious therapeutic targets. PLoS One. 2015:10:e0124254.

71. Bleedorn JA, Greuel EN, Manley PA, Schaefer SL, Markel MD, Holzman G, et al. Synovitis in dogs with stable stifle joints and incipient cranial cruciate ligament rupture: A cross-sectional study. Vet Surg. 2011;40:531-43.

72. Little JP, Bleedorn JA, Sutherland BJ, Sullivan R, Kalscheur VL, Ramaker MA, et al. Arthroscopic assessment of stifle synovitis in dogs with cranial cruciate ligament rupture. PLoS One. 2014;9:e97329.

73. Muir P, Kelly JL, Marvel SJ, Heinrich DA, Schaefer SL, Manley PA, et al. Lymphocyte populations in joint tissues from dogs with inflammatory stifle arthritis and associated degenerative cranial cruciate ligament rupture. Vet Surg. 2011:40:753-61.

74. Haynes MK, Hume EL, Smith JB. Phenotypic characterization of inflammatory cells from osteoarthritic synovium and synovial fluids. Clin Immunol. 2002 105:315-25.

75. Goldberg VM, Burstein A, Dawson M. The influence of an experimental immune synovitis on the failure mode and strength of the rabbit anterior cruciate ligament. J Bone Joint Surg Am. 1982;64:900-6. 
76. Lohmander LS, Englund PM, Dahl LL, Roos EM. The long-term consequence of anterior cruciate ligament and meniscus injuries. Am J Sports Med. 2007; 35:1756-69.

77. Paschos N. Anterior cruciate ligament reconstruction and knee osteoarthritis. World J Orthop. 2017;8:212-7.

78. Simon D, Mascarenhas R, Saltzman BM, Rollins M, Bach BR Jr, MacDonald P. The relationship between anterior cruciate ligament injury and osteoarthritis of the knee. Adv Orthop. 2015;2015:928301.

79. DeChiara TM, Kimble RB, Poueymirou WT, Rojas J, Masiakowski P, Valenzuela DM, et al. Ror2, encoding a receptor-like tyrosine kinase, is required for cartilage and growth plate development. Nat Genet. 2000;24:271-4.

80. den Hollander W, Ramos YF, Bomer N, Elzinga S, van der Bredden R, Lakenberg $\mathrm{N}$, et al. Transcriptional associations of osteoarthritis-mediated loss of epigenetic control in articular cartilage. Arthritis Rheumatol. 2015;67: 2108-16.

81. Maeda K, Kobayashi Y, Udagawa N, Uejara S, Ishihara A, Mizoguchi T, et al. Wnt5a-Ror2 signaling between osteoblast-lineage cells and osteoclast precursors enhances osteoclastogenesis. Nat Med. 2012;18:405-12.

82. Holley RJ, Tai G, Williamson AJ, Taylor S, Cain SA, Richardson SM. Comparative quantification of the surfaceome of human multipotent mesenchymal progenitor cells. Stem Cell Reports. 2015;4:473-88.

83. Song YJ, Li G, He JH, Guo Y, Yang L. Bioinformatics-based identification of microRNA-regulated and rheumatoid arthritis-associated genes. PLoS One. 2015;10:e0137551.

84. Rebagay G, Yan S, Liu C, Cheung NK. ROR1 and ROR2 in human malignancies: potentials for targeted therapy. Front Oncol. 2012;2:34

85. Ford CE, Qian Ma SS, Quadir A, Ward RL. The dual role of the novel Wnt receptor tyrosine kinase, ROR2, in human carcinogenesis. Int J Cancer. 2013; 133:779-87.

86. Yang J, Zeng J, Goddard ME, Wray NR, Visscher PM. Concepts, estimation, and interpretation of SNP-based heritability. Nat Genet. 2017;49:1304-11.

87. Nishikimi A, Uruno T, Duan X, Cao Q, Okamura Y, Saitoh T, et al. Blockade of inflammatory responses by a small-molecule inhibitor of the rac activator DOCK2. Chem Biol. 2012;19:488-97.

\section{Ready to submit your research? Choose BMC and benefit from:}

- fast, convenient online submission

- thorough peer review by experienced researchers in your field

- rapid publication on acceptance

- support for research data, including large and complex data types

- gold Open Access which fosters wider collaboration and increased citations

- maximum visibility for your research: over $100 \mathrm{M}$ website views per year 